\title{
Mimicking water striders' legs superhydrophobicity and buoyancy with cabbage leaves and nanotube carpets
}

\author{
Emiliano Lepore \\ Department of Structural, Geotechnical and Building Engineering, Laboratory of Bio-inspired Nanomechanics \\ “Giuseppe Maria Pugno", Politecnico di Torino, 10129 Torino, Italy \\ Mauro Giorcelli \\ Department of Applied Science and Technology, Politecnico di Torino, 10129 Torino, Italy \\ Chiara Saggese \\ Chemistry, Material and Chemical Engineering Department "Giulio Natta” (CMIC), Politecnico di Milano, \\ 20133 Milano, Italy \\ Alberto Tagliaferro \\ Department of Applied Science and Technology, Politecnico di Torino, 10129 Torino, Italy \\ Nicola Pugno ${ }^{\text {a) }}$ \\ Department of Civil, Environmental and Mechanical Engineering, University of Trento, I-38123 Trento, Italy
}

(Received 2 August 2012; accepted 25 October 2012)

In this work, we have studied the superhydrophobicity and buoyancy of two types of nanostructured surfaces: the cabbage leaf and a vertically aligned carbon nanotubes (VACNTs) carpet. The wettability of these surfaces were characterized by contact angle, tilting angle, sliding volume and sliding speed measurements. The results were correlated to the related surface topologies, which were investigated by scanning electron microscopy. Buoyancy of different surfaces has been investigated through measurements of the forces acting on the surface. Finally, we demonstrate that cabbage leaves and VACNT carpets have some common features with the water strider's leg, better understanding the mechanisms of buoyancy related to the structural shape and size of natural or artificial nanostructures.

\section{INTRODUCTION}

Nowadays, nature represents the main source of inspiration for scientists to develop new innovative technological materials, which are useful to daily life, such as superhydrophobic and self-cleaning surfaces ${ }^{1-5}$ or superadhesive materials. ${ }^{6}$ This approach is called "biomimicry," which "describes a process in which the ideas and concepts developed by nature are taken and implemented into technology."7

There are different types of natural surfaces providing a huge variety of functions, such as the self-cleaning effect of the Lotus leaf (Nelumbo nucifera) ${ }^{8,9}$ and the water repellency of the water strider's leg (Gerris remigis). ${ }^{10,11}$ These natural structures might be useful models to design new targets and to study how to functionalize surfaces, ${ }^{12}$ such as artificial superhydrophobic surfaces, which play a significant role in self-cleaning, for drag reduction and energy conservation. ${ }^{13,14}$

In nature, one of the crucial surface properties that has attracted great interest in biomimetic research is water

\footnotetext{
a) Address all correspondence to this author.

e-mail: nicola.pugno@unitn.it

DOI: $10.1557 /$ jmr.2012.382
}

repellency. Wettability is mainly related to the following surface factors: chemical composition, surface topology which determines the hierarchical micro- and nanostructures $^{6,15-19}$ the free surface energy ${ }^{1,20-22}$ and the anisotropic arrangement of surface structures, which could be found e.g., in the rice leaf. ${ }^{23,24}$

Superhydrophobic and self-cleaning surfaces with high static contact angle (CA) and low CA hysteresis (defined as the difference between the advancing and receding $\mathrm{CA}$ ) are characterized by the cooperation between the surface chemical compositions of low free energy and topographic micro- and nanostructures. These elements minimize water adhesion and reduce the tilting angle (TA). Furthermore, it was demonstrated that these hydrophobic surfaces, with structures at different length scales, support considerable load on a water surface, as inspired by floatation of insects on water due to their superhydrophobic legs. ${ }^{25,26}$ As a matter of fact, the special ability of water striders to walk quickly on the water surface and the superhydrophobicity of their legs, which are connected to the nanostructured surface microstructure, had attracted considerable attention in the scientific community. ${ }^{27-29}$

In this paper, two hierarchical hydrophobic surfaces, natural or artificial, with surface structures similar to those 
of the water strider's leg (G. remigis), are compared in terms of wettability and floating capability. They are the upper (adaxial) and lower (abaxial) sides of the natural cabbage leaf of Brassica oleracea ${ }^{9,30}$ and the hydrophobic side of a vertically aligned carbon nanotubes (VACNTs) carpet. ${ }^{31}$ VACNTs carpets are interesting hydrophobic surfaces for various applications, including for mimicking the lotus effect, due to their mechanical strength combined with their low density. ${ }^{12}$ Since the cabbage leaf is a hierarchical sculptured surface, because of its convex papillose epidermal cells and its three-dimensional (3D) epicuticular waxes, ${ }^{32}$ it is used as a model of an unwetted (superhydrophobic) and dirtfree (self-cleaning) surface. ${ }^{33}$ In literature, several attempts of reproducing the hierarchical architecture of cabbage leaves with densely packed nanoplates and nanoribbons on superhydrophobic surfaces, or by treating and processing an inorganic material such as copper, have been done. ${ }^{33}$ At the same time, few water strider-like models with large and stable loading capacity were fabricated using the previous superhydrophobic surfaces with the shape of a wire, a mesh, or a foil. ${ }^{26-28}$

To understand the effect of the morphology on surface wetting properties, this paper develops a surface characterization in terms of wettability through measures of CA, TA, sliding volume (SV), and sliding speed (SS), as defined in previous papers, ${ }^{21,34}$ and through field emission scanning electron microscopy (FESEM) micrographs. The aim was to observe and to discuss their superficial morphologies, which are similar to the surface of the water strider's leg. An experimental and theoretical analysis of the floating and overturning behavior of the natural cabbage leaf and the hydrophobic side of VACNTs carpet on a water surface is finally presented.

\section{MATERIALS AND METHODS}

\section{A. Surfaces production and preparation}

We consider two different nanostructured surfaces: the cabbage B. oleracea and the self-standing VACNTs carpet. The cabbage leaf was picked from a young greenhouse plant, while the millimeter-thick VACNTs carpet $(\sim 1.4 \mathrm{~mm})$ was grown on a silicon substrate using the chemical vapor deposition (CVD) technique. This process starts from a natural and ecological precursor (camphor) and a catalyst (Ferrocene, Sigma-Aldrich, St. Louis, MO); at the end of the process, the grown carpet is mechanically detached from the silicon substrate using a razor blade. This process permits to obtain self-standing mats, ${ }^{31}$ easy to manipulate, which we have used for our measurements. Wettability behavior, FESEM micrographs, and floating measurements were studied on untreated surfaces. On the other hand, wettability and floating measurements for the surface, that we have characterized with FESEM, of the water strider's leg are taken from literature. ${ }^{35}$

\section{B. Surface characterization}

Cabbage surfaces (Fig. 3) and VACNTs carpet (Fig. 4) were analyzed by FESEM (ZEISS SUPRA 40, ZEISS, Oberkochen, Germany) equipped with a field emission tungsten cathode. Samples of $\sim 0.5 \mathrm{~cm}^{2}$ were fixed to aluminum stubs by double sided adhesive carbon conductive tape (Nisshin EM Co., Ltd., Tokyo, Japan) and air dried. Samples of cabbage have been $\mathrm{Cr}$ coated $(\sim 10 \mathrm{~nm}$ in thickness), while VACNTs carpet was analyzed after detaching from silicon substrate.

These surfaces were compared to the water strider's leg (Fig. 3), which were analyzed by FESEM (FEI-Inspect ${ }^{\mathrm{TM}}$ F50, FEI, Hillsboro, OR), equipped with a field emission tungsten cathode at $2 \mathrm{kV}$. Samples of the water strider's leg were amputated from adult specimens and maintained in $70 \%$ ethanol solution. Afterward, samples were dehydrated at ambient temperature and atmospheric pressure for 12-h before analyzing under the eye of the scanning electron microscope. Thus, they were fixed to aluminum stubs by double sided adhesive carbon conductive tape (Nisshin EM Co., Ltd.). Samples were Au-Pd coated and were $\sim 25 \mathrm{~nm}$ in thickness.

\section{Measurements of wettability}

The wettability of the cabbage leaf and the hydrophobic side of VACNTs carpet were determined by measuring the static CA of distilled water droplets over samples, fixed to a horizontal plane by a soft adhesive to keep samples flat. A series of 20 (instead of 5 as in Refs. 24, 36 , and 37) random volume drops, gently deposited on surfaces with a standard single use syringe, were considered. The CA was recorded with a digital photo camera (OLYMPUS MJU 1010, OLYMPUS, Tokyo, Japan) measured and statistically analyzed with the software ImageJ 1.410.

Two distinct procedures followed to investigate the dry sliding friction and wear behavior of surfaces: (i) fixing the volume of the drop $(\sim 18 \mu \mathrm{L}$; the diameter of the spherical droplet was $\sim 2.2 \mathrm{~mm}$ ) and measuring the tilted angle of the sample stage at drop TA or (ii) fixing the angle of the specimen stage vertically $\left(90^{\circ}\right)$ and measuring the minimum SV of the drop for sliding with a $2 \mu \mathrm{L}$ volume increment. Referring to the second procedure, the SS of the drop was also determined, measuring the time of the minimum SV drop needed to cover the fixed distance of $10 \mathrm{~mm}$.

Figure 1 schematically shows the step-by-step process to determine the two additional parameters: SV and SS.

\section{Measurements of floating}

Floating behavior of the cabbage leaf and hydrophobic side of VACNTs carpet were investigated using an experimental apparatus, which was able to measure the maximal loading capacity of these surfaces before sinking (Fig. 2). 
A circular plastic bin with a diameter of $\sim 5.7 \mathrm{~cm}$ was filled with distilled water. Two considered samples, the fresh cabbage leaf of $0.089 \mathrm{~g}\left(1.75 \times 1.2 \mathrm{~cm}^{2}\right)$ and the VACNTs carpet of $0.201 \mathrm{~g}\left(1.6 \times 1.3 \mathrm{~cm}^{2}\right)$, were placed on the water without piercing the surface itself. A series of plummets (mass of $\sim 0.09 \mathrm{~g}$ each) were placed into a plastic cap $(0.195 \mathrm{~g})$, located on the side of the samples opposite to the studied surface, until the sample onset sinking. The maximum weight supported by the surfaces was determined gently and symmetrically adding plummets on the surface and evaluating the number, so the weight, which each surface sustained before piercing the water surface. This easy, replicable, and inexpensive approach could obviously be used to investigate other nanostructured surfaces.

\section{RESULTS AND DISCUSSION}

\section{A. Surface characterization}

As Figs. 3, 4, and 5 show, some likenesses are present on nanostructured surfaces of the cabbage leaf, the hydrophobic side of VACNTs carpet and water strider's leg.

Both the nanostructured surfaces of the cabbage leaf and the hydrophobic side of VACNTs carpet have nanoprotuberances that decrease the fraction of solid in contact with the liquid. This nanostructure helps the liquid re-

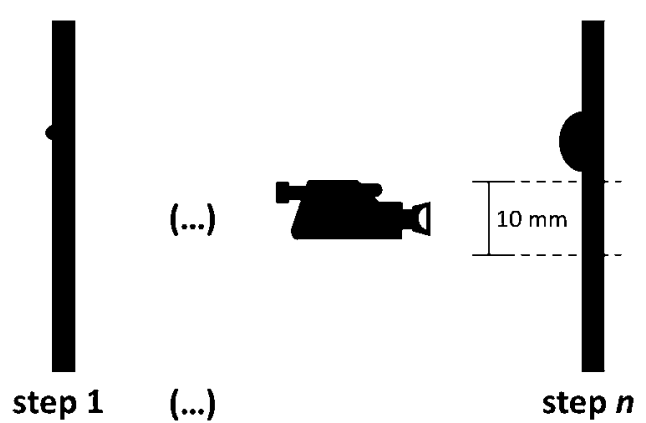

FIG. 1. The step-by-step process used to determine the two additional parameters: SV and SS. The specimen stage was fixed vertically $\left(90^{\circ}\right)$ and the drop volume was increased with $2 \mu \mathrm{L}$ droplets at each step: so $2 \mu \mathrm{L}$ drop volume at step $1,4 \mu \mathrm{L}$ drop volume at step 2 , and so on, up to the minimum sliding drop volume (SV), which is necessary to cause the sliding of the drop ( $\operatorname{step} n$ ). At step $n$, the drop starts to slide, and the SS was determined measuring the time to cover the distance of $10 \mathrm{~mm}$ (mean velocity). pulsion and thus increases the hydrophobicity. This is measurable with the CA. Moreover, plant natural surfaces present natural waxy crystals, which are automatically regenerated in case of damage by natural life cycle. Their presence increases the water repulsion and permits the phenomena of the water drop rolling.

In the water strider's legs (Fig. 5), similar hierarchical micro- and nanostructures, present in the cabbage leaf, are shown. Both, combined with waxy coatings, are responsible for the observed high water repellence.

For water-repellent and superhydrophobic surfaces, it is critically important to maintain a stable Cassie-Baxter state. ${ }^{16}$ In contrast with Wenzel state, ${ }^{15}$ which forecasts a drop conformal with the surface roughness, Cassie-Baxter regime considers a drop shape not conformal with the surface topology, thanks to the presence of air pockets. No multiscale topography is considered in the Cassie-Baxter equation namely, $\cos \vartheta_{\mathrm{C}}=\phi_{1} \cos \vartheta_{\mathrm{Y}, 1}+\phi_{2} \cos \vartheta_{\mathrm{Y}, 2}$, where $\vartheta_{\mathrm{C}}$ is the apparent $\mathrm{CA}, \phi_{1}$ is the fraction of liquid contacting the solid, $\phi_{2}$ is the fraction of liquid contacting the air, $\vartheta_{\mathrm{Y}, 1}$ is the Young CA of the liquid on the solid, and $\vartheta_{\mathrm{Y}, 2}$ is the Young CA of the liquid in air (equal to $180^{\circ}$ ). Only in the Cassie-Baxter regime, a relatively weak adhesion between the water droplet and the surface is present, but several destabilizing factors are responsible for the transition to the Wenzel regime, such as evaporation. In nature, water repellency and superhydrophobicity are obtained by different micro- and nanometer length scales to maintain the composite interface stability (Figs. 3-5). In general, natural waterrepellent and superhydrophobic plants show multiscale structures and/or structures with several hierarchical levels $(N)$. For instance, they are considered in the hierarchical law, ${ }^{6}$ $\cos \theta_{\mathrm{F}}^{(N)}=\phi_{\mathrm{S}}^{N}\left(\cos \theta_{\mathrm{Y}}+1\right)-1$, where $\theta_{\mathrm{F}}^{(N)}$ is the Fakir CA at level $N, \phi_{\mathrm{S}}^{N}$ is the solid pillar fraction at the level $N$, and $\theta_{\mathrm{Y}}$ is the Young CA of the liquid on the solid, as for the Wenzel and Cassie-Baxter models. Thus, the surface patterning at multiple length scales minimizes (or maximizes for intrinsically hydrophilic surfaces) the adhesion of liquids and particles, leading to a self-cleaning behavior.

\section{B. Measurements of wettability}

The four studied parameters (CA, SA, SV, and SS) were used for a wettability surface characterization. ${ }^{2,5,21,34,37}$
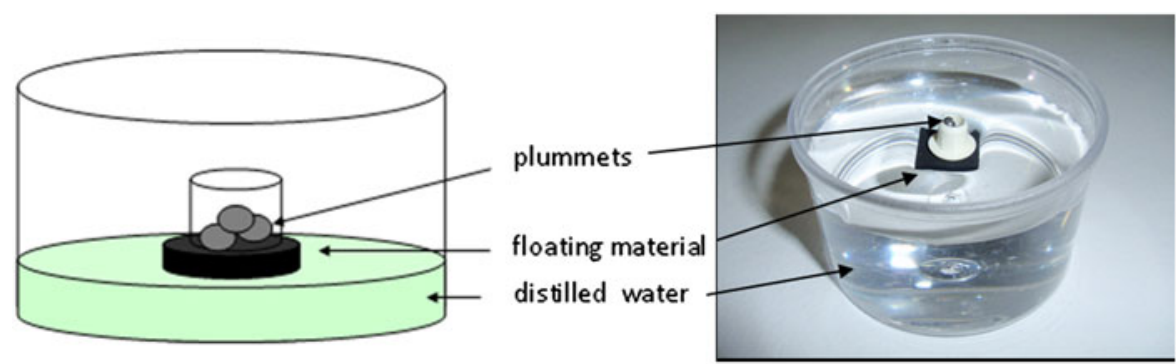

FIG. 2. Schematic view and real optical image of the load carrying setup used for floating measurements. 


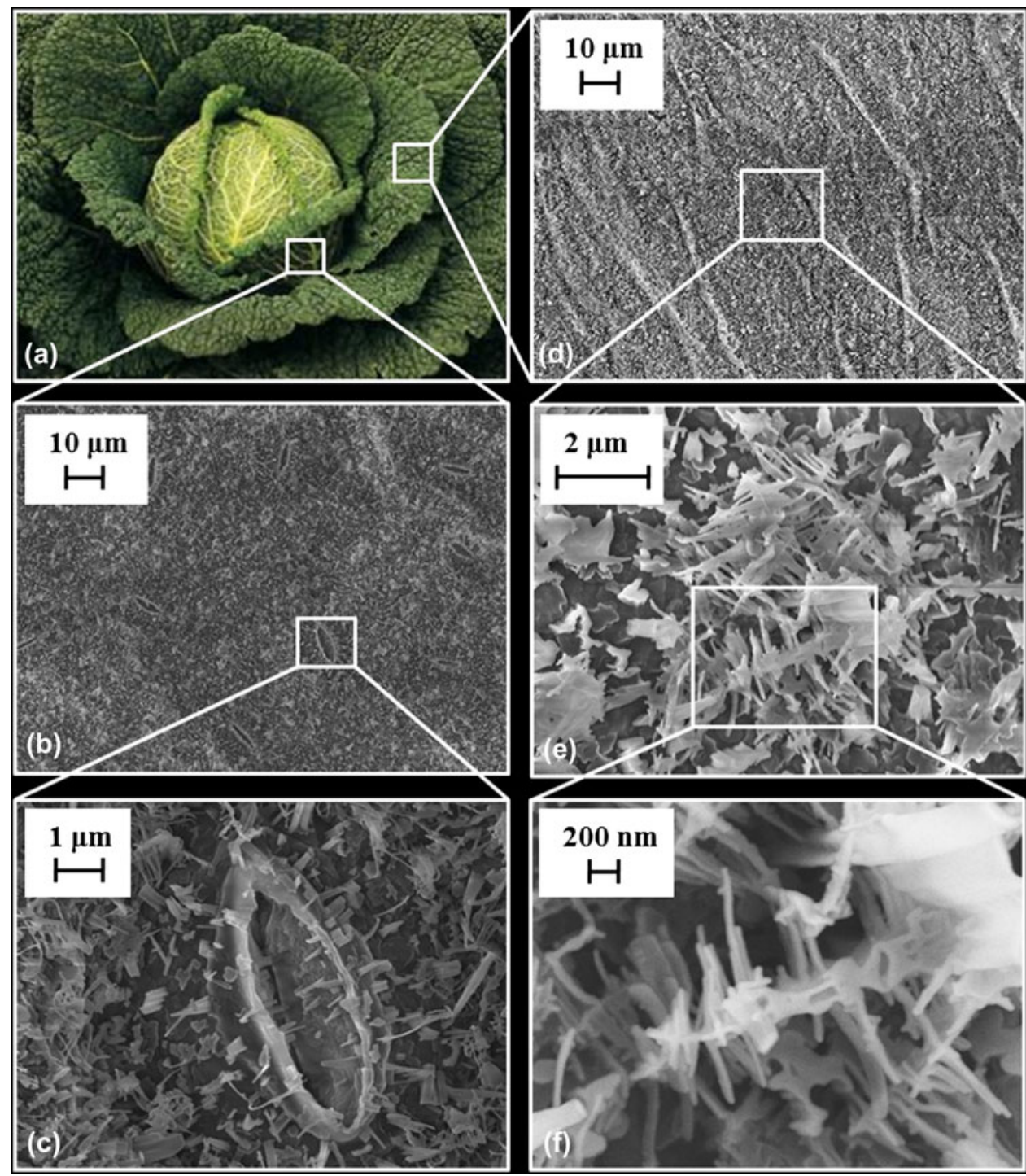

FIG. 3. (a) Two-level hierarchical structure of the cabbage leaves (B. oleracea), which consists of epidermal cells at the microscale and a superimposed layer of hydrophobic wax crystals at the nanoscale. (b, c) Stomata of the lower (L, abaxial) side of the cabbage leaf; (d, e, f) tabular and smooth cells with a dense arrangement of $3 \mathrm{D}$ wax crystals on the upper (U, adaxial) side of the cabbage leaf. ${ }^{9}$ Their width is in the range from 70 to $90 \mathrm{~nm}$.

As Fig. 6 shows, the CA values of natural and artificial surfaces are all close to $\sim 145^{\circ}$. This angle is characteristic of high hydrophobic surfaces, but only few measurements on the upper side of the cabbage leaf show a superhydrophobic behavior $\left(\mathrm{CA}>150^{\circ}\right)$.

The natural cabbage surface has a low SA. In particular, in this study, the lower side of the cabbage leaf presents the lower value of SA. Even if SA values of the artificial surface (VACNTs) are not constant in all regions, their average value $\left(\sim 45^{\circ}\right)$ remains larger than the value found for the natural surface. The particular waxy layer, which exists on natural surfaces, works as a low free energy coating and permits an easier removal of particulate depositions (self-cleaning).
When the sample stage is closer to vertical position $\left(\sim 90^{\circ}\right)$, the $\mathrm{SV}$ is almost the same for the hydrophobic side of VACNTs and the upper side of the cabbage leaf. The lower side of the cabbage leaf shows a SV value of $14 \mu \mathrm{L}$, more than double than that of the other two surfaces. A lower volume represents an improved hydrophobic behavior of the surface because the droplet needs less weight (drop volume) to slide down over the surface. In this case, the best result was reached by the upper side (U, adaxial) of the cabbage leaf.

The SS parameter on the natural surfaces results to be higher than that of the artificial surfaces, so the friction on the cabbage leaf is lower than that of the VACNTs surfaces. 


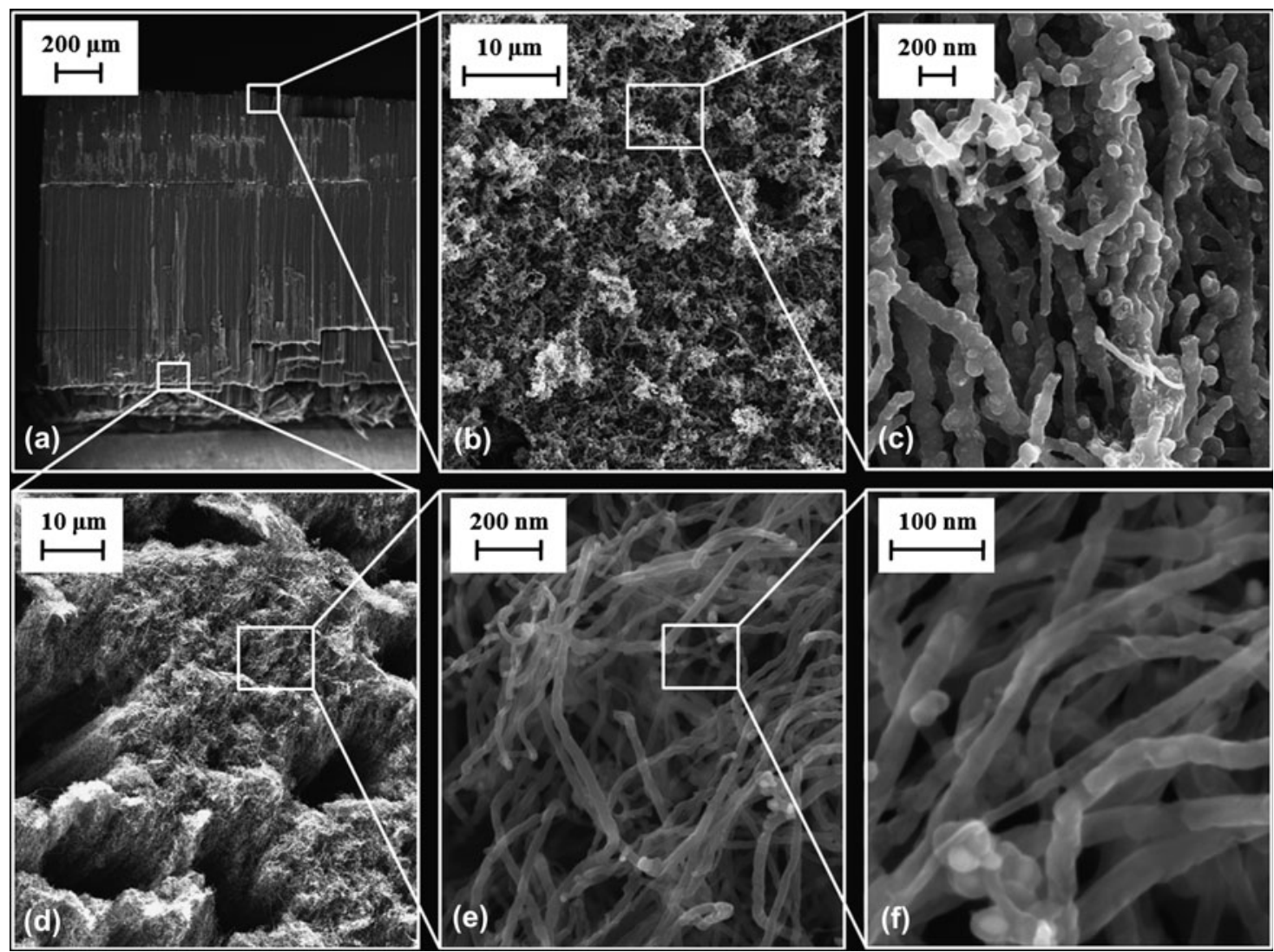

FIG. 4. (a) FESEM images of VACNTs carpet; (b, c) the hydrophobic side of the carpet, which represents the end of CNTs growing, is formed by different substances, probably amorphous carbon, and it is composed by not aligned nanotubes; (d, e, f) the other side of the carpet, attached on a silica substrate, where CNTs began to rise. They are aligned and their width is in the range from 20 to $60 \mathrm{~nm}$.

\section{Measurements of floating}

Floating measurements results are shown in Table I. Some values were rejected by using the Chauvenet's criterion. ${ }^{38}$ Maximal loading capacity data for each surface were recorded with experimental tests, as discussed in Sec. II. D.

\section{Buoyancy mechanism}

The supporting force $\left(F_{\mathrm{s}}\right)$, against no dipping, is the sum of two components: the buoyancy force $\left(F_{\mathrm{b}}\right)$ and the curvature force $\left(F_{\mathrm{c}}\right)$ :

$$
F_{\mathrm{s}}=F_{\mathrm{b}}+F_{\mathrm{c}} .
$$

The force $F_{\mathrm{b}}$ is proportional to the mass of the displaced fluid, which is calculated as the volume of fluid (water), multiplied by its density $(\rho)$. The considered volume is larger than the object volume $(b h D)$ due to the presence of the dimple $d^{39}$ as shown in Fig. 7. Accordingly:

$$
F_{\mathrm{b}} \approx \rho b h(D+d) g .
$$

The force $F_{\mathrm{c}}$ can be obtained by integrating the pressure curvature over the sample area or equivalently the vertical component of the surface tension $(\sigma \cos \theta)$ along the contact perimeter $(2 b+2 h)$ :

$$
F_{\mathrm{c}} \cong(2 b+2 h) \sigma \cos \theta \text {. }
$$

In this formula, $\sigma$ and $\theta$ are the water surface tension (for distillated water $0.07575 \mathrm{~N} / \mathrm{m}$ ) and the $\mathrm{CA}$, respectively.

Therefore, the total supporting force $F_{\mathrm{s}}$ for each surface can be expressed by:

$$
F_{\mathrm{s}}=F_{\mathrm{b}}+F_{\mathrm{c}} \cong \rho b h(D+d) g+(2 b+2 h) \sigma \cos \theta \text {. }
$$

Obviously, for buoyancy, we have:

$$
F_{\mathrm{s}}>M g
$$

It should be noted that the vertical depth of dimples $(d)$ depends on the surface CA, as established by Jiang et al., ${ }^{35}$ which analyzed the water strider leg and showed the following Eq. (2):

$$
\theta=\frac{\pi}{2}+\arctan (\sigma d)+\arcsin (2 \sigma d / D)
$$




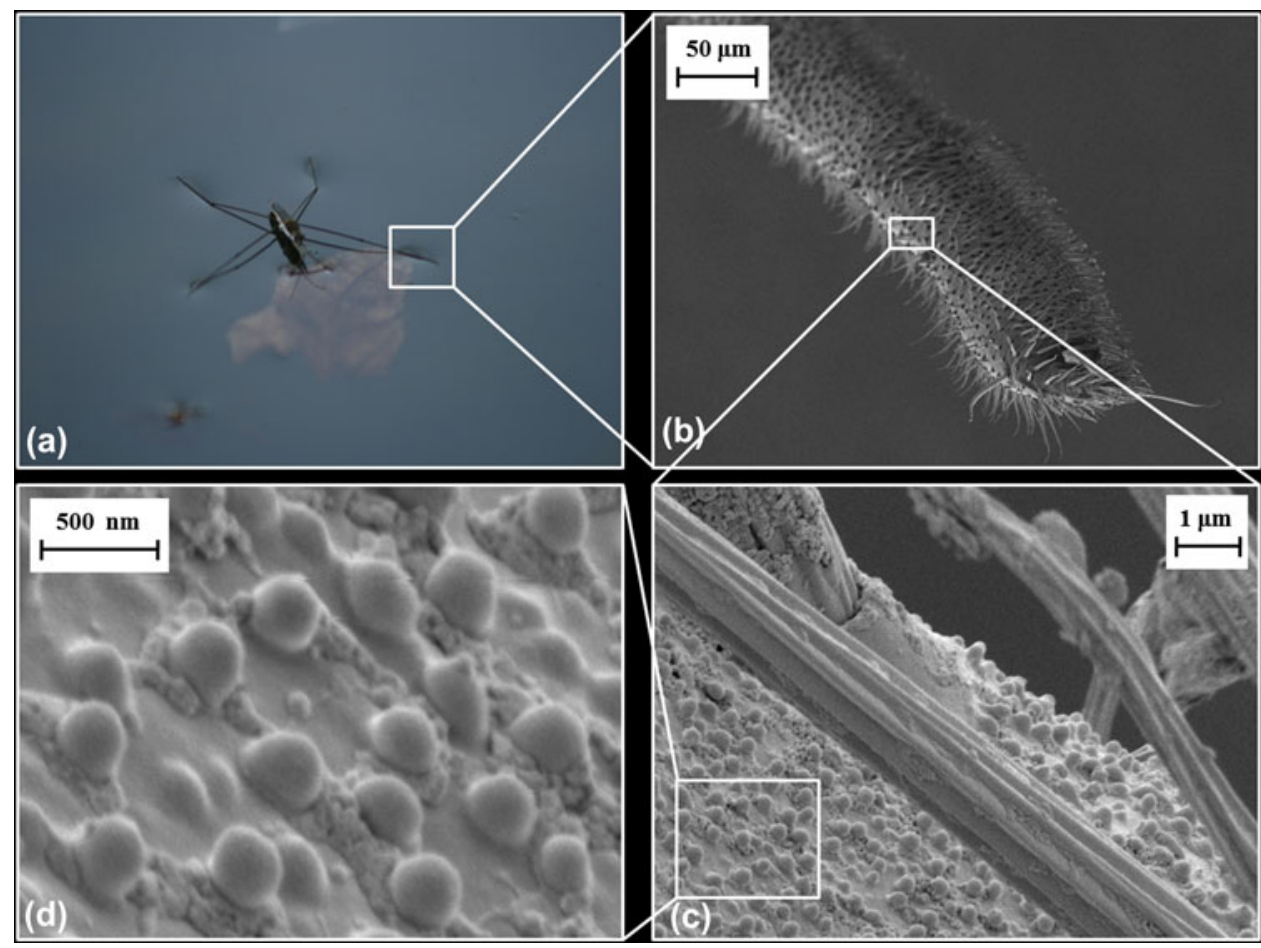

FIG. 5. Two-level hierarchical structure of the water strider's leg, which exhibits microstructures with fine nanoscale grooves under the FESEM eye: (a) a G. remigis insect, which is floating in a pond, (b) the water strider's frontal leg, which is covered by numerous oriented needle-shaped microsetae, (c) the nanogrooves of a microseta, which is composed of several papillae (d).
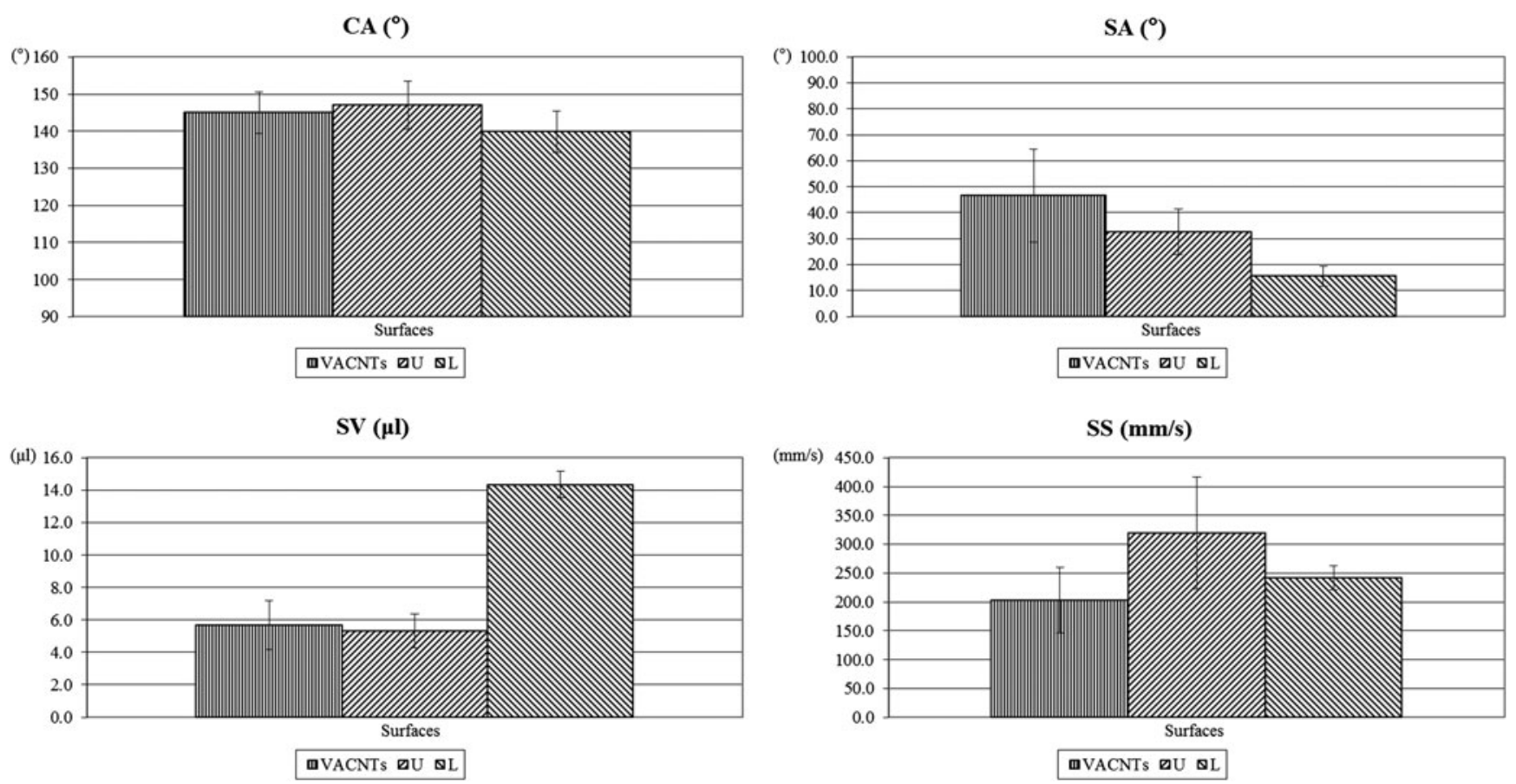

FIG. 6. The measurements of wettability for the hydrophobic side of VACNTs, the upper (U, adaxial) and lower (L, abaxial) sides of the natural cabbage leaf of B. oleracea.

As shown in Eq. (4), the mechanism of floating depends on the object size $(b, h, D)$. If we consider large objects, $F_{\mathrm{b}}$ is prevalent on $F_{\mathrm{c}}$, vice versa at small size scales. The curvature force plays a significant role when the object size is comparable or smaller than the capillary length (that for water is $2.7 \mathrm{~mm}$ ). 
Table II summarizes the contribution of $F_{\mathrm{c}}$ and $F_{\mathrm{b}}$ to the supporting force $F_{\mathrm{s}}$ for the analyzed surfaces as well as the total critical masses ( $W_{\mathrm{TOT}}$ ) and the critical depth $(d)$ of the dimples.

Calculations show that $F_{\mathrm{b}}$ is the most important factor of the total supporting force and it accounts for more than $80 \%$ for the VACNTs carpet, which has a millimeter thickness, and more than $70 \%$ for both leaf sides, which are thinner than the carpet.

Our results confirm a correlation between surfaces' loading capacities and their wettability and superhydro-

TABLE I. Results of floating experiments on two different surfaces: the natural leaf of B. oleracea and the hydrophobic side of VACNTs carpet.

\begin{tabular}{|c|c|c|c|}
\hline \multirow[b]{2}{*}{ Test } & \multicolumn{3}{|c|}{$\begin{array}{c}\text { Applied weight }(\mathrm{mg}) \text { on rectangular } \\
\text { surface }\left(2.1 \mathrm{~cm}^{2}\right)\end{array}$} \\
\hline & Leaf upper & Leaf lower & $\begin{array}{l}\text { VACNTs } \\
\text { hydrophobic }\end{array}$ \\
\hline 1 & 500.4 & 700.7 & 404.3 \\
\hline 2 & 505.1 & 605.0 & 288.7 \\
\hline 3 & 407.8 & 498.7 & 389.3 \\
\hline 4 & 406.6 & 502.9 & 391.3 \\
\hline 5 & $\ldots$ & 501.3 & $\ldots$ \\
\hline 6 & $\ldots$ & 500.7 & $\ldots$ \\
\hline 7 & $\ldots$ & 691.3 & $\ldots$ \\
\hline $\operatorname{Mean}^{\mathrm{a}}$ & 455 & 572 & 395 \\
\hline $\mathrm{SD}(\sigma)^{\mathrm{a}}$ & 55 & 93 & 54 \\
\hline Mean SD $\left(\sigma^{\prime}=\sigma / \sqrt{ } \mathrm{N}\right)^{\mathrm{a}}$ & 28 & 35 & 27 \\
\hline
\end{tabular}

${ }^{\mathrm{a}}$ Results round down using instrumental error $( \pm 0.1 \mathrm{mg})$.

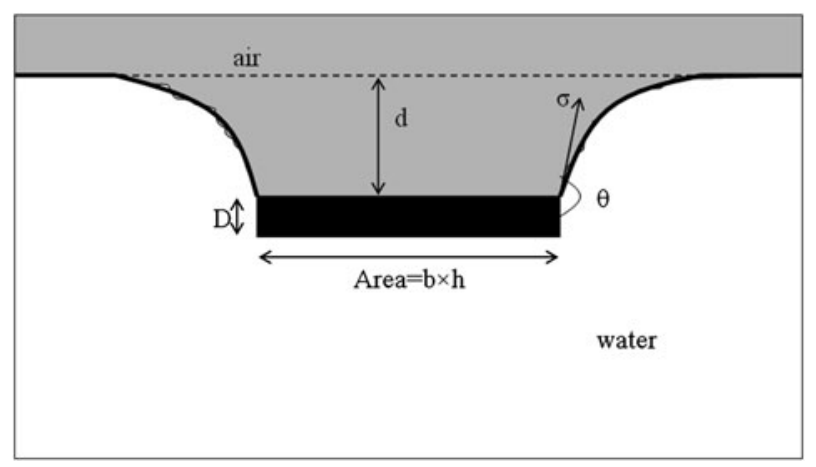

FIG. 7. Schematic illustration for the dimples of the water buoyancy model.

TABLE II. The floating behavior of each surface of $\sim 2.1 \mathrm{~cm}^{2}$ : the total supporting force and its components, the total critical load, which is applied to each surface, and the related dimples.

\begin{tabular}{lccc}
\hline \hline & Leaf upper & Leaf lower & VACNTs hydrophobic \\
\hline$F_{\mathrm{c}} / g(\mathrm{mg})$ & 0.238 & 0.281 & 0.247 \\
$F_{\mathrm{b}} / g(\mathrm{mg})$ & 0.861 & 0.798 & 1.934 \\
$F_{\mathrm{s}} / g(\mathrm{mg})$ & 1.099 & 1.079 & 2.181 \\
$W_{\mathrm{TOT}}(\mathrm{mg})$ & 706.6 & 884.4 & 730.6 \\
Dimple $(\mathrm{mm})$ & 3.5 & 3.2 & 7.9 \\
\hline \hline
\end{tabular}

phobicity. The maximum weight supported by $2.1 \mathrm{~cm}^{2}$ was obtained by the L side of the cabbage leaf and it is equal to $691 \mathrm{mg}$ or $\sim 3 \mathrm{~kg} / \mathrm{m}^{2}$. This result underlines the higher bearing capacity of the natural surface (in particular, the L side of cabbage leaf), if compared to the artificial surface (VACNTs carpet).

Another interesting aspect is the ratio between the weight of the support (cabbage leaf and VACNTs) and the supporting weight. In our case, the weight of cabbage leaf is $89 \mathrm{mg}$, while the weight of VACNTs is $200 \mathrm{mg}$. They support $\sim 5$ times their weight in the case of natural surface, while $\sim 2$ in the case of artificial surface, about 3 times the Archimedes' prediction. This result is confirmed by the value reported in Table II. A single insect's leg can withstand a force up to $\sim 15$ times the weight of the insect's body without piercing the water surface and move a volume of water roughly 300 times larger than that of the leg itself. ${ }^{35}$

It was also observed that the wettability of the surfaces strongly affected the depth of the dimples and the loading capacity of the surfaces. Moreover, loading capacity increase with increasing CA was observed.

\section{CONCLUSIONS}

Learning from nature allows us to create new innovative devices and to provide useful guidelines for engineering new materials with a range of possible industrial applications.

In this article, we have analyzed and compared two types of nanostructured surfaces: the cabbage leaf of B. oleracea (biological surface) and a VACNTs carpet (artificial surface). We have observed that the hierarchical arrangement of structures of the surface have a strong impact on the macroscopic observable surface wettability. The presence of surface roughness at multiple length scales is necessary to increase the wetting resistance and the water floating capacity, as water striders' legs show.

In conclusion, we have demonstrated that the cabbage leaf and the VACNTs carpet have some common features with the water strider's legs: the carrying load on water, the superhydrophobic property and the nanoand microstructures of the surface.

The best result in terms of wettability and floating behavior is given by the cabbage leaf and this may orient the new research to optimize the cabbage-like morphologies and performances of the VACNTs carpet in terms of wettability for desiging new nanofluidic systems water strider inspired.

\section{ACKNOWLEDGMENTS}

The authors would like to thank E. Enrico, "Nanofacility Piemonte," INRIM Institute, and S. Guastella, Department of Applied Science and Technology, Politecnico di Torino 
for the fundamental help performing the FESEM micrographs. The authors thank Gianluca Ferrari for the final English grammar supervision. This work is supported by the ERC Ideas Starting Grant 2011 No. 279985 "BIHSNAM, Bio-inspired Hierarchical Super-Nanomaterials," which is gratefully acknowledged by the PI NMP.

\section{REFERENCES}

1. T. Sun, L. Feng, X. Gao, and L. Jiang: Bioinspired surfaces with special wettability. Acc. Chem. Res. 38(8), 644 (2005).

2. B. Bhushan and Y.C. Jung: Wetting, adhesion and friction of superhydrophobic and hydrophilic leaves and fabricated micro/ nanopatterned surfaces. J. Phys. Condens. Matter 20(24), 225010 (2008).

3. M. Nosonovsky and B. Bhushan: Superhydrophobic surfaces and emerging applications: Non-adhesion, energy, green engineering. Curr. Opin. Colloid Interface Sci. 14(20), 270 (2009).

4. B. Bhushan, Y.C. Jung, and K. Koch: Micro-, nano- and hierarchical structures for superhydrophobicity, self-cleaning and low adhesion. Philos. Trans. R. Soc. London, Ser. A 367(41), 1631 (2009).

5. K. Koch, B. Bhushan, and W. Barthlott: Multifunctional surface structures of plants: An inspiration for biomimetics. Prog. Mater. Sci. 54(41), 137 (2009).

6. N.M. Pugno: Towards a Spiderman suit: Large invisible cables and self-cleaning releasable superadhesive materials. J. Phys. Condens. Matter 19(17), 395001 (2007).

7. M. Ayre: Biomimicry - A Review, Work Package Report (European Space Research \& Technology Centre, European Space Agency, Noordwijk, The Netherlands, 2003).

8. W. Barthlott: Epidermal and seed surface characters of plants: Systematic applicability and some evolutionary aspects. Nord. J. Bot. 1(10), 345 (1981).

9. C. Neinhuis and W. Barthlott: Characterization and distribution of water-repellent, self-cleaning plant surfaces. Ann. Bot. 79(10), 667 (1997).

10. J.W.M. Bush and D.L. Hu: Walking on water: Biolocomotion at the interface. Annu. Rev. Fluid Mech. 38(40), 339 (2006).

11. J.W.M. Bush, D.L. Hu, and M. Prakash: The integument of waterwalking arthropods: Form and function. Adv. Insect Physiol. 34(72), 117 (2008).

12. L. Feng, S. Li, Y. Li, H. Li, L. Zhang, J. Zhai, Y. Song, B. Liu, L. Jiang, and D. Zhu: Super-hydrophobic surfaces: From natural to artificial. Adv. Mater. 14(3), 1857 (2002).

13. A. Nakajima, K. Hashimoto, and T. Watanabe: Recent studies on super-hydrophobic films. Monatsh. Chem. 132(10), 31 (2001).

14. Y.C. Jung and B. Bhushan: Mechanically durable carbon nanotube composite hierarchical structures with superhydrophobicity, selfcleaning, and low-drag. ACS Nano 3(8), 4155 (2009).

15. R.N. Wenzel: Resistance of solid surfaces to wetting by water. Ind. Eng. Chem. 28(6), 988 (1936).

16. A.B.D. Cassie and S. Baxter: Wettability of porous surfaces. Trans. Faraday Soc. 40(5), 546 (1944).

17. D. Quéré: Wetting and roughness. Ann. Rev. Mater. Res. 38(28), 71-99 (2008).

18. Y. Su, B. Ji, K. Zhang, H. Gao, Y. Huang, and K. Hwang: Nano to micro structural hierarchy is crucial for stable superhydrophobic and water-repellent surfaces. Langmuir 26(5), 4984 (2010).

19. M. Nosonovsky and B. Bhushan: Hierarchical roughness optimization for biomimetic superhydrofobic surfaces. Ultramicroscopy 107(10), 969 (2007).
20. T. Nishino, M. Meguro, K. Nakamae, M. Matsushita, and Y. Ueda: The lowest surface free energy based on -CF3 alignment. Langmuir 15(3), 4321 (1999).

21. E. Lepore and N. Pugno: Superhydrophobic polystyrene by direct copy of a lotus leaf. BioNanoScience 1(7), 136 (2011).

22. A. Nakajima, K. Abe, K. Hashimoto, and T. Watanabe: Preparation of hard super-hydrophobic films with visible light transmission. Thin Solid Films 376(3), 140 (2000).

23. Z. Guo and W. Liu: Biomimic from the superhydrophobic plant leaves in nature: Binary structure and unitary structure. Plant Sci. 172(9), 1103 (2007).

24. T. Sun, G. Wang, H. Liu, L. Feng, L. Jiang, and D. Zhu: Control over the wettability of an aligned carbon nanotube film. J. Am. Chem. Soc. 125(3), 14996 (2003).

25. H. Jin, M. Kettunen, A. Laiho, H. Pynneonen, J. Paltakari, A. Marmur, O. Ikkala, and R.H.A. Ras: Superhydrophobic and superoleophobic nanocellulose aerogel membranes as bioinspired cargo carriers on water and oil. Langmuir 27(4), 1930 (2011).

26. Q. Pan, J. Liu, and Q. Zhu: A water strider-like model with large and stable loading capacity fabricated from superhydrophobic copper foils. App. Mat. Inter. 2(4), 2026 (2010).

27. X. Zhang, J. Zhao, Q. Zhu, N. Chen, M. Zhang, and Q. Pan: Bioinspired aquatic microrobot capable of walking on water surface like a water strider. ACS Appl. Mater. Interfaces 3(6), 2630 (2011).

28. X. Wu and G. Shi: Production and characterization of stable superhydrophobic surfaces based on copper hydroxide nanoneedles mimicking the legs of water striders. J. Phys. Chem. B 110(5), 11247 (2006).

29. L. Jiang, X. Yao, H. Li, Y. Fu, L. Chen, Q. Meng, W. Hu, and L. Jiang: Water strider legs with a self-assembled coating of single-crystalline nanowires of an organic semiconductor. Adv. Mater. 22(3), 376 (2010).

30. K. Koch, B. Bhushan, and W. Barthlott: Diversity of structure, morphology and wetting of plant surfaces. Soft Matter 4(20), 1943 (2008).

31. M. Pavese, S. Musso, S. Bianco, M. Giorcelli, and N. Pugno: An analysis of carbon nanotube structure wettability before and after oxidation treatment. J. Phys. Condens. Matter 20(7), 474206 (2008).

32. K. Koch, H.F. Bohn, and W. Barthlott: Hierarchically sculptured plant surfaces and superhydrophobicity. Langmuir 25(4), 14116 (2009).

33. J. Liu, X. Huang, Y. Li, Z. Li, Q. Chi, and G. Li: Formation of hierarchical $\mathrm{CuO}$ microcabbages as stable bionic superhydrophobic materials via a room-temperature solution-immersion process. Solid State Sci. 10(8), 1568 (2008).

34. E. Lepore, P. Faraldi, L. Boarino, and N. Pugno: Plasma and thermoforming treatments to tune the bio-inspired wettability of polystyrene. Composites Part B 43(9), 681 (2012).

35. X. Feng, X. Gao, Z. Wu, L. Jiang, and Q. Zheng: Superior water repellency of water strider legs with hierarchical structures: Experiments and analysis. Langmuir 23(4), 4892 (2007).

36. S.M. Lee, I.D. Jung, and J.S. Ko: The effect of the surface wettability of nanoprotrusions formed on network-type microstructures. J. Micromech. Microeng. 18(7), 125007 (2008).

37. J. Yeo, M.J. Choi, and D.S. Kim: Robust hydrophobic surfaces with various micropillar arrays. J. Micromech. Microeng. 20(8), 025028 (2010).

38. S.M. Ross: Peirce's criterion for the elimination of suspect experimental data. J. Eng. Technol. 20(3), 38 (2003).

39. F. Shi, J. Niu, J. Liu, F. Liu, Z. Wang, X. Feng, and X. Zhang: Towards understanding why a superhydrophobic coating is needed by water striders. Adv. Mater. 19(4), 2257 (2007). 